\title{
Effects of neuromuscular taping on muscular strength, range of motion and pain intensity in the glenohumeral joint in professional handball athletes: blinded randomized clinical trial
}

\author{
Cristiane Rissatto Jettar Lima', Paulo Fernandes Pires', Charlini Simoni Hartz¹, Ester Moreira de Castro², \\ Elisa Bizetti Pelai ${ }^{3}$, Delaine Rodrigues Bigaton ${ }^{4}$.
}

\begin{abstract}
Background: Recent investigations in handball athletes point to the need to carry out rehabilitation programs that aim to improve the instability of the glenohumeral joint, widely used in the various tasks of this modality. Objectives: To evaluate the immediate and short-term effects of a neurofunctional elastic bandage (NEB) on muscular strength, range of motion and pain intensity in the glenohumeral joint in handball athletes with shoulder dysfunction. Method: This study was composed of 20 professional male handball athletes who presented shoulder pain at rest and dysfunction of the shoulder, divided into two groups; experimental ( $n=10)$ and placebo $(n=10)$. Before and one hour after the application of NEB the following were evaluated: maximal isometric muscular strength during movements of the glenohumeral joint (load cell), the range of motion (ROM) of the glenohumeral joint (fleximetry), pain intensity in the shoulder at rest and during movement, and the pressure pain threshold (algometry). NEB was maintained for 72 hours in all volunteers, during which a diary of shoulder pain was recorded for the short-term assessment. We used the ANOVA two-way repeated measures considering the possibility of group by time interaction, adopting a $5 \%$ level for significant differences. The treatment effect size was analyzed by means of Cohen's d values. Results: There was no group by time interaction for any of the variables $(p>0.05)$, however, there was a large effect of the treatment for reducing pain in the experimental group after the application of NEB in the short-term period $(\mathrm{d}=0.83$ ). Conclusion: There were no significant effects on muscular strength, range of motion or pain intensity in the shoulder in handball athletes immediately after implementation of NEB.
\end{abstract}

Keywords: Physiotherapy; Athletic Injuries; Shoulder; Handball.

\section{INTRODUCTION}

The practice of handball involves the performance of high power gestures and intense body contact associated with repetitiveness of action. Among the most common sporting gestures, we highlight the large number of passes, throws followed by jumps, and rapid changes of direction ${ }^{(1)}$. On average, each player performs about 48,000 pitches at a speed of about $130 \mathrm{~km} / \mathrm{h}$ per season ${ }^{(2)}$, which justifies the high prevalence of disorders related to the upper limbs in these athletes. ${ }^{(3)}$

The involvement of dysfunctions in the glenohumeral joint in athletes is commonly seen in sports that require the performance of movements with arms above the head and repetitive characteristics, such as handball, volleyball and baseball ${ }^{(2-4)}$. These disorders are triggered by microinjuries generated by repetitiveness and may result in prejudice to performance of the sport gesture and hence athlete performance. The most common alterations found are the onset of pain, shoulder instability, scapular dyskinesia( ${ }^{(1)}$, an increased range of motion (ROM) of external rotation and a reduction in ROM of internal rotation of the glenohumeral joint in the dominant shoulder performing the throwing gesture ${ }^{(5,6)}$.

Recent investigations in handball athletes point to the need to carry out rehabilitation programs that aim to improve the instability of the glenohumeral joint, widely used in the various tasks of this modality ${ }^{(7,8)}$. Among the intervention methods aimed at minimizing instability, the neuromuscular elastic bandage (NEB) stands out, widely used in various areas of rehabilitation and especially in the sports community in various modalities ${ }^{(8,9)}$.

Although studies that tested the effectiveness of NEB on dysfunction in athletes present controversies, recent research has indicated positive effects in the short term period on reducing pain and increasing the ROM of the glenohumeral joint and cervical spine ${ }^{(10-14)}$, and improved performance in

Corresponding author: Delaine Rodrigues Bigaton Av. Jaime Pereira, 3701 - Ondas, Piracicaba/SP - CEP 13403800 Email: drodrigues@unimep.br, Phone: (19) 31241558

${ }^{4} \mathrm{PhD}$ in Biology, Dental Oral Pathology and professor of the Graduate Program in Human Movement Sciences at the Universidade Metodista de Piracicaba UNIMEP - Piracicaba (SP), Brazil 
sports that require efforts with repetitive movement and high intensity characteristics ${ }^{(9,15-17)}$. Additionally, the method is described as capable of generating improvement in local circulation, a reduction in edema, facilitation or relaxation of muscles, and improving joint position and proprioception due to optimization of the sensory mechanism ${ }^{(7-8,18)}$, characteristics which contribute to improving athletic performance.

Williams et al. ${ }^{(19)}$ in a systematic review, studied the effects of NEB in the prevention and treatment of sports injuries, and found a major limitation in the results of the studies analyzed, since the majority presented inadequate intervention design and few used a specific population of athletes. Thus, we emphasize the importance of this study and the need for further research in the population of athletes, considering the specifics of the handball sport.

In this context, considering the previously documented effects of NEB and no studies evaluating the effects of the direct application of NEB's on the glenohumeral joint in handball athletes, the aim of this study was to evaluate the immediate and short-term effects of NEB on muscular strength, ROM, pain intensity in the shoulder at rest and during movement and the shoulder pressure pain threshold in handball athletes with shoulder dysfunction. The hypothesis was that the application of NEB would generate increased muscular strength, increase ROM in the glenohumeral joint (mainly internal rotation movement) and reduce the pain intensity in handball athletes with shoulder dysfunction.

\section{METHODS}

\section{STUDY DESIGN}

This study was treated as a blind randomized clinical trial. Note that only the volunteers were blinded to the treatment received. The participants were randomized through block random draw (1: 1) into two groups: experimental group (EG) and placebo group (GP).

The recruitment of volunteers, data collection and analysis were performed at the Sports Physiotherapy Department, XV de Piracicaba Club, from August to September 2014. This study was approved by the Research Ethics Committee of the Methodist University of Piracicaba (UNIMEP) under protocol number $73 / 13$. All volunteers signed a free and informed consent.

\section{PARTICIPANTS}

The study included 20 professional handball players, male, members of the Handball Sports Association ${ }^{(15)}$, aged between 17 and 35 years $(22.1 \pm 4.72)$. Were included athletes with shoulder pain at rest, confirmed using a diary of pain through a visual analog pain scale (VAS) for 7 consecutive days; dysfunction in the shoulder, according to the Disability Arm Shoulder Hand questionnaire (DASH); and the absence of cervical disfunction, evaluated through the Disability
Index (NDI). Volunteers who were on drug therapy were excluded from the study (analgesics, anti-inflammatories and muscle relaxants); as well as those who had undergone surgical procedures in the shoulder and/or neck.

\section{INTENSITY OF PAIN}

The intensity of pain in the shoulder was evaluated at rest and during the general movement of the glenohumeral joint (flexion, extension, abduction, horizontal adduction, external rotation, and internal rotation) using a Visual Analog Scale (VAS) ${ }^{(20)}$.

\section{RANGE OF MOTION}

The maximum range of motion (ROM) of the glenohumeral joint of the painful shoulder $\left({ }^{\circ}\right)$ was evaluated using a fleximeter, Sanny ${ }^{\circledR}$ (Sanny, São Paulo, Brazil, L- 6010), in flexion, extension, abduction, horizontal adduction, external rotation, and internal rotation. Two evaluation attempts were recorded for each movement and the average of the attempts retained for further analysis.

\section{PRESSURE PAIN THRESHOLD}

The pressure pain threshold was measured using an algometer, brand $\operatorname{Kratos}^{\circledR}$ model DDK 200, (Kratos Equipments, São Paulo, Brazil) gradually applying a constant pressure to the deltoid muscle (middle portion) and descending part of the trapezius muscle of the painful shoulder. Volunteers remained seated in a chair, with the torso upright, leaning back, feet flat on the floor and hands resting on the legs. For the evaluation of the deltoid muscle, gradual pressure was applied at the midpoint between its origin and insertion, and for the descending part of the trapezius, constant gradual pressure was applied at half the distance between the spinous process of the 7th cervical vertebra and the acromion of the scapular ${ }^{(21)}$. The evaluator exercised gradual compression perpendicular to the muscle fibers until the volunteer reported any intensity of pain, at which point this value was recorded. If the volunteer felt no pain, compression was terminated when it reached the maximum threshold of $4 \mathrm{Kgf}^{(22)}$. Each item was rated twice with a 1 minute interval between compressions, and the order of each assessed muscle was selected randomly for each volunteer. For further processing the average value was used in $\mathrm{Kg} / \mathrm{F}$ in each of the 2 muscle compression points.

\section{MUSCULAR STRENGTH}

Muscular strength was evaluated through maximal isometric contraction ( $\mathrm{Kg} / \mathrm{F}$ ) of the muscle groups involved in the movements of flexion, extension, horizontal adduction, abduction, external rotation and internal rotation of the glenohumeral joint of the painful shoulder, using a load cell, $\operatorname{Kratos}^{\circledR}(\mathrm{MM}-100)$, connected to an EMG signal data acquisition module, EMG System Brasil ${ }^{\circledR}$ model EMG 830 C. (EMG System. 
do Brasil Ltda, São José dos Campos, SP, Brasil). For each movement, the volunteers were positioned standing in front of a concrete column, which was attached to the load cell by a leather strap, with the aim of providing stability to perform the movements. All subjects were instructed to stand with the lower limb contralateral to the evaluated shoulder anteriorly (anterior feint) in order to avoid compensation in the pelvis and trunk. Two maximal isometric contraction repetitions were performed of 5 second duration, with an interval of 30 seconds between contractions, and the order of the evaluated movements was randomly selected for each volunteer. For the processing of muscular strength data, the average of the maximum values obtained through two repetitions of each analyzed movement was used for analysis.

\section{Neuromuscular Elastic Bandage}

The bandage used for the intervention was the Kinesiology ${ }^{(3)}$ Tape (WETAPE Inc, Seoul, Korea). The EG received the application of NEB, with pressure to the deltoid muscle (anterior, middle and posterior fibers) and the descending part of the trapezius muscle of the painful shoulder, associated with application for multi-axial instability ${ }^{(23)}$. The GP received the application of NEB without pressure only to the distal portion of the deltoid muscle of the painful shoulder ${ }^{(24)}$. The exact pressure of the NEB used during the research was based on a pilot study in the engineering laboratory of the UNIMEP campus Santa Barbara D'Oeste, where, by means of a load cell, the bandage was stretched up to the point of rupture and subsequently the length of the bandage at $50 \%$ and $20 \%$ of the breaking point was established in a standardized manner. It should be noted that to maintain the standardization of the bandage, an anchor (end of bandage held without pressure) of two centimeters was always maintained.

In the GE, subjects remained seated with the torso upright and feet supported. For application of the NEB on the deltoid muscle anterior and posterior fibers, the bandage was cut in the form of a " $Y$ ", with the intention of grouping the deltoid muscle ${ }^{(23)}$, and for the medium fibers a bandage was used in the form of an "I". Application began with the setting of the base of the " $Y$ " tape just below the deltoid tuberosity of the humerus, leaving two centimeters of tape without tension. After fixation, the tape anterior and posterior to the " $Y$ ", together with the "I ", were applied to the elongated deltoid muscle at $20 \%$ pressure, following the application methods described below, as shown in Figure 1 .

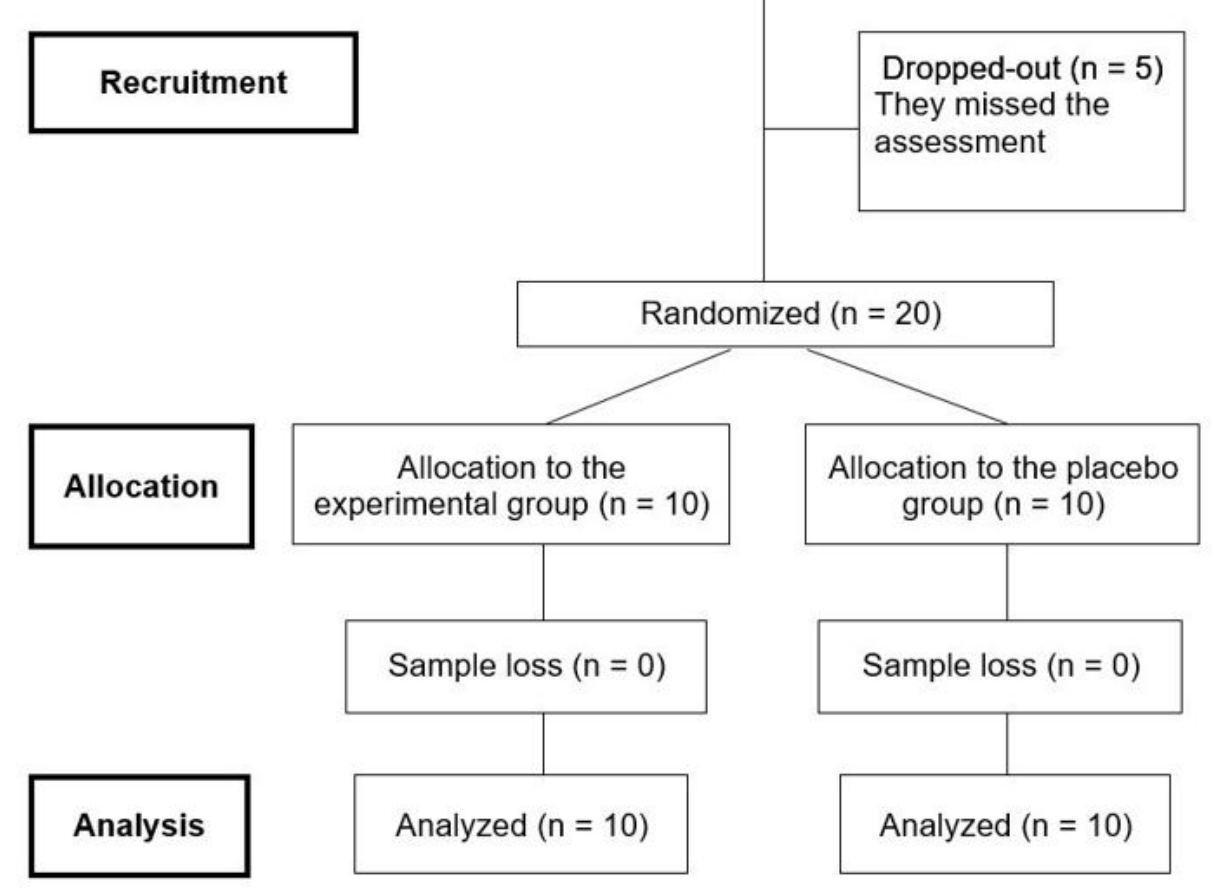

Figure 1. Flowchart of participants during the study 
Application to the deltoid muscle anterior fibers: the volunteer's arm was positioned in horizontal abduction at 90 degrees with external rotation and extension of the glenohumeral joint. The anterior of the " $Y$ " was applied along the outer margin of the deltoid muscle (anterior fibers) toward the acromion - clavicular joint, with the last two centimeters of tape being left without tension.

Application to the deltoid muscle posterior fibers: the volunteer's arm was positioned in horizontal adduction with internal rotation of the glenohumeral joint. The posterior of the " $Y$ " was applied along the outer margin of the deltoid muscle (posterior fibers) toward the acromion - clavicular joint, with the last two centimeters of tape being left without pressure.

Application to the deltoid muscle middle fibers: We used the tape in the "I" format. The volunteer's arm was positioned next to the trunk at rest and the cervical spine positioned in flexion, with lateral and rotation inclination to the opposite side of the application of the bandage. Continuity was provided by fixing the base of the "I" just below the deltoid humeral tuberosity, continuing the application along the path of the deltoid (middle fibers) and trapezius (descending part) to the spinous process of the seventh cervical vertebra, with the last two centimeters of both tape ends being left without tension. Application for Multiaxial Instability: The volunteer conducted and maintained an abduction of the glenohumeral joint at $90^{\circ}$. For this application, another tape in the shape of an "I" was fixed with $50 \%$ pressure. One end was fixed immediately below the acromion-clavicular joint, allowing $2 \mathrm{~cm}$ of tape without tension, and the other end was fixed just below the spine of the scapula, also being fixed leaving $2 \mathrm{~cm}$ without tension ${ }^{(23)}$, as shown in Figure 2 (A).

In the GP, volunteers remained seated with the torso upright, feet supported and upper limbs alongside the body. A bandage in the shape of an "I" was used, $10 \mathrm{~cm}$ long, without applied pressure, to the distal portion of the deltoid muscle transversaly ${ }^{(4)}$, as shown in Figure 2 (B).

\section{PROCEDURES}

After meeting the established eligibility criteria, the research included 3 evaluation moments: 1 ) pre-intervention evaluation: the intensity of pain was evaluated at rest and during general movement of the glenohumeral joint, ROM, pressure pain threshold and muscular strength of the glenohumeral joint. 2) Immediately post-evaluation: one hour after the intervention, the pain intensity ratings, range of motion, pressure pain threshold and muscular strength of the glenohumeral joint were evaluated again. 3) Short-term rating (72 hours): at the end of the post-immediate evaluation,
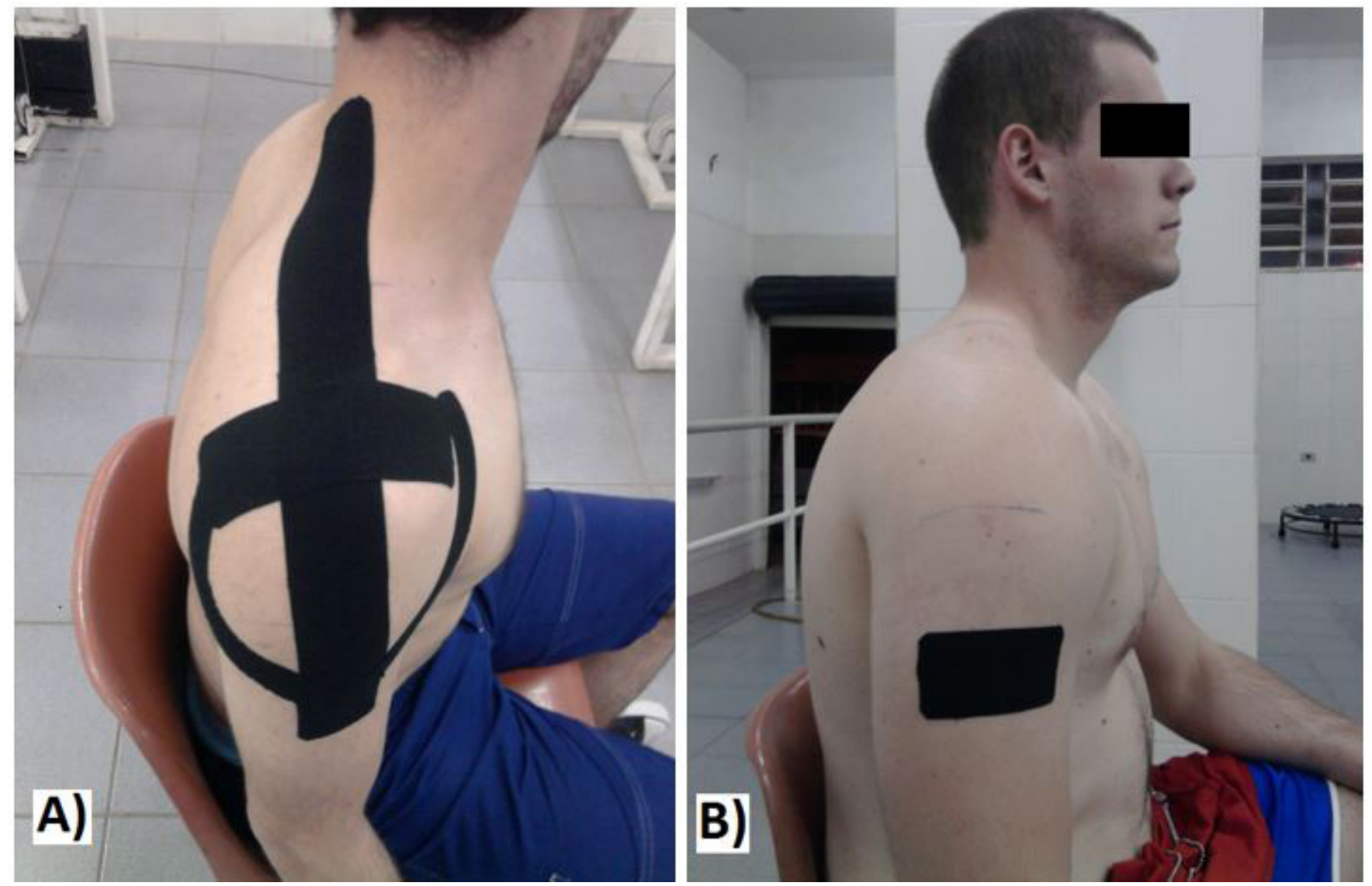

Figure 2. Application of neuromuscular elastic bandage. (A) experimental group, (B) placebo group. 
the volunteers were again given a back pain diary to register their shoulder pain at rest for 3 consecutive days, at night. The subjects were told to keep the bandages on for 72 hours. In the case of the bandage partially coming off, they were advised to remove it completely and record the removal date. It is worth mentioning that the experimental procedure was applied after two months of training and one month of the competitive period, for both groups. The training periodization consisted of five weekly night sessions, including three tactical-technical, and two physical and a weekly game at the weekend.

\section{Calculation of Sample Size}

The sample size in the present study was based on a pilot study. The strength value obtained during maximal isometric contraction of abduction of the glenohumeral joint ( $E G=11.53 \pm 2.57 ; G P=14.03 \pm 2.85$ ) was used as the outcome variable, which suggested 20 volunteers. The sample size calculation was performed using the BioEstat ${ }^{\circledR}$ application, version 5.0, (Belém (PA), Brazil) 2007, an alpha level of 5\% and $80 \%$ power for independent samples.

\section{Data analysis}

Initially, the normality of the data was tested using the Shapiro-Wilk test. For comparison of the data the two-way ANOVA test for repeated measures was used for each dependent variable of the study. The time factor (pre and post-intervention) was used as within-subject factor and group (experimental and placebo) as between-subject. The hypothesis of interest was the interaction group by time. We also used the Student $t$ test for intergroup comparison of the pre-intervention period, the anthropometric characteristics (age, BMI) as well as the variables used as criteria for inclusion in this study. For the analysis of intra-rater reliability the intraclass correlation coefficient was calculated $\left(\mathrm{ICC}_{3,1}\right)$. The values of reliability were considered as follows; "Iow reliability" ( $<0.40)$, "good reliability" $(\geq 0.40$ and $\leq 0.75)$ and "excellent reliability" (>0.75) ${ }^{(25)}$. The standard error of measurement (SEM) was also calculated using the formula. The significance level used for analysis of all the statistical tests described was 5\%, applied via SPSS 17.0 (Chicago, IL USA). The intra-group clinical treatment effect size was assessed using the Cohen's $d$ test for all the dependent variables. For the calculation, the division value of the average difference between each evaluation period of each group by the pooled standard deviation was taken into account. The " $\mathrm{d}$ " established values were "low treatment effect" ( $\leq 0.2)$, "moderate treatment effect" ( $\cong 0.5)$ and "high treatment effect $(\geq 0.8)^{\text {"(26) }}$. The minimum detectable changes (MMD) of the dependent variables were calculated for comparison of pre and post intervention with the values of treatment effect size. The formula used for the calculation was .

\section{RESULTS}

The eligibility criteria for this study were applied to 25 previously recruited male athletes. There was a sample loss of 5 volunteers during the evaluations due to non-attendance. Finally, the remaining athletes were randomly allocated to the groups according to the flowchart in Figure 1.

In the pre-intervention period homogeneity was observed for the anthropometric characteristics, age and BMI between groups ( $p>0.05$ ), respectively: $23 \pm 6.13$ and $24.83 \pm 3.09$ for the EG and $20.89 \pm 3.21$ and $25.78 \pm 3.11$ for the GP. In the scores from the DASH and NDI questionnaires, homogeneity between the groups was also found $(p>0.05)$, respectively: $23.76 \pm 14.49$ and $4.70 \pm 3.90$ for the EG and $36.83 \pm 20.65$ and $5.20 \pm 2.71$ for the GP.

There was excellent intra-rater reliability to evaluate the maximum strength of isometrics for all movements of the glenohumeral joint (ICC $: 0.79$ to 0.93 and SEM: 2.75 to 5.84 ), excellent reliability for ROM evaluation of the glenohumeral joint (ICC 30.92 to 0.99 and SEM: 2.93 to 6.87) and excellent reliability for evaluation of the pressure pain threshold (ICC ${ }_{3,1}: 0.85$ to 0.87 and SEM: from 0.32 to 0.45 ).

The analysis of muscular strength during maximal isometric contraction, observed in Table 1, there was no significant time by group interaction for flexion ( $F=2.67, p=0.11)$, extension $(\mathrm{F}=0.33, p=0.57)$, abduction $(\mathrm{F}=0.09, p=0.75)$, horizontal adduction ( $F=1.63, p=0.21)$, external rotation $(F=0.58, p=0.45)$ or internal movements $(F=0.17, p=0.68)$. In the treatment effect size a moderate effect was observed only in the maximum isometric twitch force in the bending movement of the glenohumeral joint for the placebo group and the MMD value found proved to be far from the actual differences after the intervention in both groups.

In the ROM analysis of the glenohumeral joint, Table 2, no significant time by group interaction was found in flexion ( $\mathrm{F}=0.06, p=0.80)$, extension $(\mathrm{F}=1.11, p=0.13)$, abduction $(F=0.008, p=0.93)$, horizontal adduction $(F=1.21, p=0.28)$, external rotation $(\mathrm{F}=1.80, p=0.19)$ or internal rotation $(\mathrm{F}=1.19$, $p=0.28)$. The treatment effect sizes observed were mild to moderate in general for the ROMs, and the MMD values proved to be far from actual differences after the intervention in both groups. In the analysis of the pressure pain threshold, Table 3, there was no significant time by group interaction for the deltoid muscles $(F=0.04, p=0.84)$ or trapezius descending part $(\mathrm{F}=0.04, p=0.83)$. There was a slight treatment effect size and expected MMD much larger than the real differences in the post-intervention. For pain intensity, Table 3, there was no significant time by group interaction for VAS at rest $(F=0.21$, $p=0.65$ ), VAS during general movement of the glenohumeral joint $(\mathrm{F}=0.74, p=0.39)$ or daily pain $(\mathrm{F}=1.94, p=0.18)$. However, there was a high treatment effect for the experimental group in the short-term period, as shown by a reduction in pain intensity assessed by daily pain. 
Table 1. Intragroup and intergroup comparison of the maximum values of strength in isometric contractions to the glenohumeral joint movements, and their treatment effects for each group.

\begin{tabular}{|c|c|c|c|c|c|}
\hline & Pre Intervention & Post Intervention & Intragroup difference & $\begin{array}{c}\text { Cohen's d } \\
\text { (Pre x Post Intervention) }\end{array}$ & MDD \\
\hline \multicolumn{6}{|c|}{ Flexion isometric contraction of the glenohumeral joint $(\mathrm{Kg} / \mathrm{F})$} \\
\hline Experimental Group & $16.89 \pm 5.46$ & $16.64 \pm 3.73$ & $-0.26(-2.23 \mid 2.74)$ & 0.06 & \multirow{2}{*}{4.14} \\
\hline Placebo Group & $15.34 \pm 3.16$ & $17.46 \pm 4.78$ & $2.12(-4.28 \mid 0.03)$ & 0.52 & \\
\hline \multicolumn{6}{|c|}{ Extension isometric contraction of the glenohumeral joint $(\mathrm{Kg} / \mathrm{F})$} \\
\hline Experimental Group & $20.3 \pm 4.81$ & $20.45 \pm 3.89$ & $0.15(-1.54 \mid 1.24)$ & 0.03 & \multirow{2}{*}{4.07} \\
\hline Placebo Group & $18.88 \pm 4.47$ & $19.52 \pm 5.21$ & $0.64(-1.96 \mid 0.68)$ & 0.13 & \\
\hline \multicolumn{6}{|c|}{ Abduction of isometric contraction of the glenohumeral joint (Kg/F) } \\
\hline Experimental Group & $13.4 \pm 3.14$ & $14.03 \pm 2.85$ & $0.63(-1.8 \mid 0.54)$ & 0.20 & \multirow{2}{*}{3.97} \\
\hline Placebo Group & $13.37 \pm 4.20$ & $13.61 \pm 5.84$ & $0.24(-2.76 \mid 2.28)$ & 0.05 & \\
\hline \multicolumn{6}{|c|}{ Isometric contraction of adduction Horizontal the glenohumeral joint (Kg/F) } \\
\hline Experimental Group & $15.5 \pm 3.58$ & $14.33 \pm 2.43$ & $-1.18(-1.17 \mid 3.52)$ & 0.38 & \multirow{2}{*}{5.28} \\
\hline Placebo Group & $14.51 \pm 4.74$ & $15.2 \pm 6.94$ & $0.69(-2.99 \mid 1.61)$ & 0.12 & \\
\hline \multicolumn{6}{|c|}{ Isometric contraction of external rotation of the glenohumeral joint $(\mathrm{Kg} / \mathrm{F})$} \\
\hline Experimental Group & $13.49 \pm 3.74$ & $13.36 \pm 3.52$ & $-0.13(-0.79 \mid 1.06)$ & 0.04 & \multirow{2}{*}{3.46} \\
\hline Placebo Group & $12.51 \pm 3.73$ & $13.1 \pm 4.87$ & $0.59(-2.51 \mid 1.34)$ & 0.14 & \\
\hline \multicolumn{6}{|c|}{ Isometric contraction of internal rotation of the glenohumeral joint $(\mathrm{Kg} / \mathrm{F})$} \\
\hline Experimental Group & $15.19 \pm 3.25$ & $15.91 \pm 3.23$ & $0.72(-2.41 \mid 0.97)$ & 0.04 & \multirow{2}{*}{3.44} \\
\hline Placebo Group & $14.54 \pm 5.27$ & $15.72 \pm 6.72$ & $1.18(-3.06 \mid 0.7)$ & 0.14 & \\
\hline
\end{tabular}

There was no significant difference in the group $x$ time interaction ( $p>0.05)$. Test used: ANOVA two-way repeated measures with Bonferroni correction. Data are expressed as mean \pm standard deviation at the study evaluation moments (pre and post - intervention), mean difference (confidence interval 95\%) for intra-group analysis, treatment effect size (Cohen's d) and minimum detectable change (MDC).

Table 2. Intragroup and intergroup comparison of ROM values of the glenohumeral joint, and their treatment effects for each group.

\begin{tabular}{|c|c|c|c|c|c|}
\hline & Pre Intervention & Post Intervention & Intragroup difference & $\begin{array}{c}\text { Cohen's d } \\
\text { (Pre x Post Intervention) }\end{array}$ & MMD \\
\hline \multicolumn{6}{|c|}{ Flexion range of motion of the glenohumeral joint $\left({ }^{\circ}\right)$} \\
\hline Experimental Group & $165.05 \pm 14.35$ & $165.6 \pm 18.43$ & $-0.55(-6.74 \mid 5.64)$ & 0.03 & \multirow{2}{*}{4.15} \\
\hline Placebo Group & $171.75 \pm 12.14$ & $171.2 \pm 12.12$ & $0.55(-7.19 \mid 8.29)$ & 0.05 & \\
\hline \multicolumn{6}{|c|}{ Extension range of motion of the glenohumeral joint $\left({ }^{\circ}\right)$} \\
\hline Experimental Group & $43.45 \pm 9.92$ & $44.35 \pm 7.94$ & $-0.9(-6.48 \mid 4.68)$ & 0.1 & \multirow{2}{*}{5.53} \\
\hline Placebo Group & $42.1 \pm 13.04$ & $39.45 \pm 17.1$ & $-2.65(-2.52 \mid 7.82)$ & 0.17 & \\
\hline \multicolumn{6}{|c|}{ Horizontal adduction range of motion of the glenohumeral joint $\left({ }^{\circ}\right)$} \\
\hline Experimental Group & $59.55 \pm 7.80$ & $62.05 \pm 10.13$ & $2.5(-7.12 \mid 2.12)$ & 0.28 & \multirow{2}{*}{7} \\
\hline Placebo Group & $63.6 \pm 17.01$ & $58.5 \pm 12.63$ & $-5.1(-9.81 \mid 20.01)$ & 0.34 & \\
\hline \multicolumn{6}{|c|}{ Abduction of motion of the glenohumeral joint $\left({ }^{\circ}\right)$} \\
\hline Experimental Group & $173.55 \pm 25.26$ & $178.25 \pm 21.1$ & $4.7(-13.39 \mid 3.99)$ & 0.2 & \multirow{2}{*}{7.43} \\
\hline Placebo Group & $171.2 \pm 14.70$ & $176.35 \pm 13.13$ & $5.15(-12.86 \mid 2.56)$ & 0.37 & \\
\hline \multicolumn{6}{|c|}{ External rotation range of motion of the glenohumeral joint $\left({ }^{\circ}\right)$} \\
\hline Experimental Group & $90.95 \pm 12.81$ & $92.55 \pm 14.70$ & $1.60(-8.30 \mid 5.10)$ & 0.12 & \multirow{2}{*}{8.92} \\
\hline Placebo Group & $88.6 \pm 12.89$ & $95.3 \pm 14.03$ & $6.7(-12.06 \mid-1.34)$ & 0.5 & \\
\hline \multicolumn{6}{|c|}{ Internal rotation range of motion of the glenohumeral joint $\left({ }^{\circ}\right)$} \\
\hline Experimental Group & $63.00 \pm 13.30$ & $66.65 \pm 11.74$ & $3.65(-8.16 \mid 0.86)$ & 0.29 & \multirow{2}{*}{9.72} \\
\hline Placebo Group & $67.75 \pm 11.22$ & $67.5 \pm 15.17$ & $-0.25(-6.45$ | 6.95$)$ & 0.02 & \\
\hline
\end{tabular}

There was no significant difference in the group $x$ time interaction ( $p>0.05)$. Test used: ANOVA two-way repeated measures with Bonferroni correction. Data are expressed as mean \pm standard deviation at the study evaluation moments (pre and post - intervention), mean difference (confidence interval $95 \%$ ) for intra-group analysis, treatment effect size (Cohen's d) and minimum detectable change (MDC). 
Table 3. Intragroup and intergroup comparison of the pain threshold values of the pressure intensity and pain in the shoulder, and their treatment effects for each group.

\begin{tabular}{|c|c|c|c|c|c|}
\hline & Pre Intervention & Post Intervention & Intragroup difference & $\begin{array}{c}\text { Cohen's d } \\
\text { (Pre x Post Intervention) }\end{array}$ & MMD \\
\hline \multicolumn{6}{|c|}{ Pain threshold pressure of the deltoid muscle $(\mathrm{Kg} / \mathrm{f})$} \\
\hline Experimental Group & $3.56 \pm 0.57$ & $3.52 \pm 0.57$ & $-0.04(-0.35 \mid 0.42)$ & 0.06 & \multirow{2}{*}{0.64} \\
\hline Placebo Group & $3.33 \pm 0.59$ & $3.24 \pm 0.52$ & $-0.09(-0.31 \mid 0.48)$ & 0.15 & \\
\hline \multicolumn{6}{|c|}{ Pain threshold pressure of the descending part of the trapezius muscle $(\mathrm{Kg} / \mathrm{F})$} \\
\hline Experimental Group & $2.94 \pm 0.47$ & $2.88 \pm 0.2$ & $-0.07(-0.22 \mid 0.36)$ & 0.19 & \multirow{2}{*}{0.46} \\
\hline Placebo Group & $2.65 \pm 0.37$ & $2.54 \pm 0.58$ & $-0.11(-0.19 \mid 0.41)$ & 0.20 & \\
\hline \multicolumn{6}{|c|}{ To rest pain intensity - VAS $(\mathrm{cm})$} \\
\hline Experimental Group & $0.54 \pm 0.94$ & $0.68 \pm 0.92$ & $0.14(-0.78 \mid 0.5)$ & 0.14 & \multirow{2}{*}{$\begin{array}{c}\text { Not } \\
\text { applicable }\end{array}$} \\
\hline Placebo Group & $1.93 \pm 2.25$ & $1.72 \pm 1.75$ & $-0.21(-1.35 \mid 1.77)$ & 0.11 & \\
\hline \multicolumn{6}{|c|}{ Pain intensity to the general movement of the glenohumeral joint - VAS $(\mathrm{cm})$} \\
\hline Experimental Group & $2.16 \pm 2.09$ & $1.39 \pm 1.77$ & $-0.77(-0.04 \mid 1.58)$ & 0.4 & \multirow{2}{*}{$\begin{array}{c}\text { not } \\
\text { applicable }\end{array}$} \\
\hline Placebo Group & $2.23 \pm 2.58$ & $2.02 \pm 1.53$ & $-0.21(-1.03 \mid 1.45)$ & 0.1 & \\
\hline \multicolumn{6}{|l|}{ Pain diary $(\mathrm{cm})$} \\
\hline Experimental Group & $2.42 \pm 2.55$ & $0.74 \pm 1.23$ & $-1.68(-0.04 \mid 3.4)$ & 0.83 & \multirow{2}{*}{$\begin{array}{c}\text { Not } \\
\text { applicable }\end{array}$} \\
\hline Placebo Group & $2.24 \pm 1.56$ & $1.72 \pm 1.5$ & $-0.52(-0.22 \mid 1.26)$ & 0.34 & \\
\hline
\end{tabular}

There was no significant difference in the group $x$ time interaction ( $p>0.05$ ). Test used: ANOVA two-way repeated measures with Bonferroni correction. Data are expressed as mean \pm standard deviation at the study evaluation moments (pre and post - intervention), mean difference (confidence interval $95 \%)$ for intra-group analysis, treatment effect size (Cohen's d) and minimum detectable change (MDC).

\section{DISCUSSION}

Regarding muscular strength of the maximum isometric contraction evaluated in this study, there were no significant differences in group by time interactions for any of the glenohumeral joint movements. The observed treatment effect size was mild to moderate for both groups. Finally, there was no significant difference in strength values after application of NEB compared with the values of the minimum detectable change.

Fu et al. ${ }^{(27)}$ corroborate the findings of the present study, since they also found no effects on muscular strength of the quadriceps and hamstrings after the application of NEB in healthy athletes, reporting that the lack of effect may have been caused by a failure in tactile stimulation generated by the NEB, damaging the modulation of muscular strength, which may also have occurred in the present study. Moreover, the absence of results related to muscular strength in the present study may be explained by the profile of the athletes, since they were high performing and possibly presented high muscular strength.

Kim et al. ${ }^{(28)}$, observed a significant effect on the peak internal rotation torque in individuals with tendinitis of the shoulder after the use of NEB. In part, the lack of results in the present study may be explained by the choice to evaluate isometric muscular strength and not isokinetic as used by Kim et al. Moreover, the authors specifically assessed individuals with tendinitis in the shoulder, unlike the present study which evaluated athletes with shoulder dysfunction (mild to moderate) selected via a disability questionnaire.
Fratocchi et al. ${ }^{(29)}$ found increased peak concentric elbow torque after application of NEB in the biceps muscle in asymptomatic individuals. In contrast, Csapo et al. ${ }^{(30)}$, emphasized in a systematic review study, that NEB is not capable of generating increased muscular strength in healthy adults.

Thus, it appears that the effects of NEB on muscular strength are still controversial, and further studies with the methodological rigor of randomized clinical trials should be conducted to add clarification.

For the other variables evaluated in this study (ROM, pressure pain threshold and intensity of shoulder pain) no significant differences, treatment effect size or appreciable differences were observed when comparing the MMD values with the pre and post-intervention differences (one hour later).

Even considering the pre-established context in which handball athletes who perform a large number of shots and passes can present an upward trend of ROM external rotation and reduction in WMD internal rotation of the dominant glenohumeral joint ${ }^{(5,6)}$ in the present study no significant difference or treatment effect was observed for increased internal rotation or reduction in external rotation at the shoulder of the athletes. It is thought that the small effect generated by NEB may be related to the high muscular fitness, together with the low level of disability and mild pain of the athletes evaluated. In assessing the short-term pain intensity through daily pain (after 3 days of application of NEB), there was a high treatment effect in the EG, with pain reduced by $1.68 \mathrm{~cm}$. 
However, the statistical analysis showed no significant difference in the group by time interaction. It is known that the analgesic effect generated by the application of NEB is the result of the exteroceptive action generated on the skin through the activation of mechanoreceptors, causing a depolarization to trigger nerve impulses along the afferent fibers to the central nervous system, resulting in regulation of pain mechanisms ${ }^{(31,32)}$. Similar results to those found in the present study were observed by Artioli and Bertolini ${ }^{(33)}$ and Kaya et al. ${ }^{(13)}$, who analyzed clinical trials of NEB on pain, noting greater effects of bandage application in the short term period.

Therefore, the results of this study found that the application of NEB did not provide significant effects, although beneficial effects of NEB in reducing short-term pain were shown, evidenced by the high treatment effect, demonstrating the first recorded effects of NEB on pain intensity in the shoulder in professional handball athletes, highlighting the method as a possible tool for complementary therapy.

This research had some limitations: 1 ) the selection of the sample with a low shoulder dysfunction score and mild pain and 2) evaluation of muscular strength only through maximal isometric contraction, as isotonic contractions could present different results to those found in this study.

\section{CONCLUSION}

In conclusion, the study hypothesis was not confirmed, since no significant differences were observed for any of the variables after the application of NEB. However, the method was presented as a possible tool to help reduce the intensity of short-term shoulder pain in professional handball athletes.

\section{AUTHOR'S CONTRIBUTION}

CRJL contributed to bibliographic review, study design, ethics application, data collection, data analysis, final writing, PFP contributed to bibliographic review, study design, ethics application, data analysis, final writing, CSH contributed to data collection, data analysis, EMC contributed to data collection, data analysis, final writing, EBP contributed to data analysis, final writing, DRB contributed to bibliographic review, study design, ethics application, data analysis, final writing.

\section{CONFLICTS OF INTEREST}

Nothing to declare

\section{AUTHOR DETAILS}

${ }^{1}$ Master in Physical Therapy at the Graduate Program in Physical Therapy, Universidade Metodista de Piracicaba- UNIMEP - Piracicaba (SP), Brazil.

${ }^{2}$ Master, Graduate Program in Human Movement Sciences at the Universidade Metodista de Piracicaba - UNIMEP - Piracicaba (SP), Brazil.

${ }^{3}$ Master in Physical Therapy at the Graduate Program in Physical Therapy, Faculdade de Ciências e Tecnologia - FCT / UNESP - Presidente Prudente (SP), Brazil.

\section{REFERENCES}

1. Myklebust G, Hasslan L, Bahr R, Steffen K. High prevalence of shoulder pain among elite Norwegian female handball players. Scand J Med Sci Sports. 2013;23(3):288-94.
2. Seil R, Rupp S, Tempelhof S, Kohn D. Sports injuries in team handball. A one-year prospective study of sixteen men's senior teams of a superior nonprofessional level. Am J Sports Med. 1998;26(5):681-7.

3. Wilk KE, Obma P, Simpson CD, Cain EL, Dugas JR, Andrews JR. Shoulder injuries in the overhead athlete. J Orthop Sports Phys Ther. 2009;39(2):3854.

4. Clarsen B, Bahr R, Heymans MW, Engedahl M, Midtsundstad G, Rosenlund $L$ et al. The prevalence and impact of overuse injuries in five Norwegian sports: Application of a new surveillance method. Scand J Med Sci Sports. 2015;25(3):323-30.

5. Dines JS, Frank JB, Akerman M, Yocum LA. Glenohumeral internal rotation deficits in baseball players with ulnar collateral ligament insufficiency. Am J Sports Med 2009;37:566-70.

6. Escamilla RF, Andrews JR. Shoulder muscle recruitment patterns and related biomechanics during upper extremity sports. Sports Med. 2009; 39:569-90.

7. Aarseth LM, Suprak DN, Chalmers GR, Lyon L, Dahlquist DT. Kinesio Tape and Shoulder-Joint Position Sense. J Athl Train. 2015;50(8):785-91.

8. Van Herzeele M, van Cingel R, Maenhout A, De Mey K, Cools A. Does the application of kinesiotape change scapular kinematics in healthy female handball players? Int J Sports Med. 2013;34(11):950-5.

9. Hsu YH, Chen WY, Lin HC, Wang WT, Shih YF. The effects of taping on scapular kinematics and muscle performance in baseball players with shoulder impingement syndrome. J Electromyogr Kinesiol. 2009;19(6):1092-9.

10. Shakeri H, Keshavarz R, Arab AM, Ebrahimi I. Clinical effectiveness of kinesiological taping on pain and pain-free shoulder range of motion in patients with shoulder impingement syndrome: a randomized, double blinded, placebo-controlled trial. Int J Sports Phys Ther. 2013;8(6):800-10.

11. González-Iglesias J, Fernández-de-Las-Peñas C, Cleland JA, Huijbregts P, Del Rosario Gutiérrez-Vega M. Short-term effects of cervical kinesio taping on pain and cervical range of motion in patients with acute whiplash injury: a randomized clinical trial. J Orthop Sports Phys Ther. 2009;39(7):515-21.

12. Garcia-Muro F, Rodríguez-Fernández AL, Herrero-de-Lucas A. Treatment of myofascial pain in the shouder with Kinesiotaping. A case report. Man Ther. 2010;15(3):292-5.

13. Kaya E, Zinnuroglu M, Tugcu I. Kinesio taping compared to physical therapy modalities for the treatment of shoulder impingement syndrome. Clin Rheumatol. 2011;30(2):201-7.

14. Taylor RL, O'Brien L, Brown T. A scoping review of the use of elastic therapeutic tape for neck or upper extremity conditions. J Hand Ther. 2014;27(3):235-45.

15. Bicici S, Karatas N, Baltaci G. Effect of athletic taping and kinesiotaping ${ }^{\circledR}$ on measurements of functional performance in basketball players with chronic inversion ankle sprains. Int J Sports Phys Ther. 2012;7(2):154-66.

16. Lee JH, Yoo WG. Treatment of chronic Achilles tendon pain by Kinesio taping in an amateur bARMinton player. Phys Ther Sport. 2012;13(2):1159.

17. Ho YH, Lin CF, Chang $\mathrm{CH}$, Wu HW. Effect of ankle kinesio taping on vertical jump with run-up and countermovement jump in athletes with ankle functional instability. J Phys Ther Sci. 2015; 27(7): 2087-2090. MC4540823.

18. Morris D, Jones D, Ryan H, Ryan CG. The clinical effects of Kinesio ${ }^{\circledR}$ Tex taping: A systematic review. Physiother Theory Pract. 2013;29(4):259-70.

19. Williams $S$, Whatman $C$, Hume PA, et al. Kinesio taping in treatment and prevention of sports injuries. A meta-analysis of the evidence for its effectiveness. Sports Med. 2012;42:153-164.

20. Ferreira-Valente MA, Pais-Ribeiro JL, Jensen MP. Validity of four pain intensity rating scales. Pain. 2011;152(10):2399-404.

21. Grossi DB, Chaves TC, Gonçalves MC, Moreira VC, Canonica AC, Florencio $\mathrm{LL}$, et al. Pressure pain threshold in the craniocervical muscles of women withepisodic and chronic migraine: a controlled study. Arq Neuropsiquiatr. 2011; 69(4):607-12. 
22. Ylinen J, Nykãnen $M$, Kautiainen $H$, Hãkkinen A. Evaluation of repeatability of pressure algometry on the neck muscles for clinical use. Man Ther. 2007;12(2): 192-7.

23. Kase K, Wallis J, Kase T. Clinical Therapeutic Applications of the Kinesio Taping Method. Tokyo: Ken Ikai Co Ltd; 2003.

24. Thelen M, Dauber J, Stoneman P. The clinical efficacy of kinesio tape for shoulder pain: a randomized, double blind clinical trial. J Orthop Sports Phys Ther. 2008; 38:389-95.

25. Weir JP. Quantifying test-retest reliability using the intraclass correlation coefficient and the SEM. J Strength Cond Res. 2005;19(1):231-40.

26. Cohen J. Statistical power analysis for the behavioral sciences. 2nd ed. New Jersey: Lawrence Erlbaum; 1988.

27. Fu CT, Wong AMK, Pei YC, Wu KP, Chou SW, Lin YC. Effect of Kinesio taping on muscle strength in athletes - A pilot study. Journal of Science and Medicine in Sport. 2008;11:198-201.
28. Kim SY, Kang MH, Kim ER, Oh JS. Kinesio Taping improves shoulder internal rotation and the external/internal rotator strength ratio in patients with rotator cuff tendinitis. Isokinet Exerc Sci. 2014;22, (3):259-263.

29. Fratocchi G, Di Mattia F, Rossi R, Mangone M, Santilli V, Paoloni M. Influence of Kinesio Taping applied over biceps brachii on isokinetic elbow peak torque. A placebo controlled study in a population of young healthy subjects. J Sci Med Sport. 2013;16(3):245-9.

30. Csapo R, Alegre LM. Effects of Kinesio ${ }^{\circledR}$ taping on skeletal muscle strength-A meta-analysis of current evidence. J Sci Med Sport. 2015;18(4):450-6.

31. Halseth T, McChesney JW, DeBeliso M, Vaughn R, Lien J. The effects of Kinesio taping on proprioception at the ankle. J Sports Sci Med. 2004; 3:1-7.

32. Akbaş E, Atay AO, Yüksel I. The effects of aditional kinesio taping over exercise in the treatment of patellofemoral pain syndrome. Acta Orthop Traumatol Turc. 2011; 45(5):335-341.

33. Artioli DP, Bertolini GRF. Kinesio taping: aplicação e seus resultados sobre a dor: revisão sistemática. Fisioter Pesq. 2014;21(1):94-99. 